\title{
OnLine ApPENDix FOR Weak States And Steady States: The Dynamics of Fiscal Capacity
}

\author{
Timothy Besley, Ethan Ilzetzki and Torsten Persson*
}

September 12, 2012.

\section{Distortionary Taxation}

This appendix outlines a more general version of the model in the main body of the paper. We augment the model to allow for tax evasion and distortionary taxes. This appendix is organized as follows. Section 1.1 outlines the general model and section 1.2 solves for its equilibrium. Sections 1.3 and 1.4 describe steady states first for a social planner and then when policy is politically-motivated. The latter gives conditions for the existence of three types of steady state: common interest, redistributive, and weak. The conditions parallel the cohesiveness and stability conditions in the body of the paper, and we show that the conditions in the benchmark model are special cases of the ones presented here. In section 1.5 we discuss how the demand for public goods affects the stability and cohesiveness conditions and the possibility of multiple equilibria in this model. Section 1.6 proves the claims made elsewhere in this appendix.

*Besley: London School of Economics and CIFAR. Ilzetzki: London School of Economics. Persson: IIES, Stockholm University and CIFAR. 


\subsection{The model}

\subsubsection{Households}

The model is identical to the benchmark model in all dimensions not discussed here. Household income is no longer exogenous. Instead, in each period $s$, households choose the hours of work they supply $h_{s}$ and have access to a technology that transforms a unit of labour into $\omega$ units of consumption. As in the benchmark model, they face a tax rate $t_{s}$, but can now evade taxation at a cost $\tau_{s}$ per unit of income concealed from tax collectors. $\tau_{s}$ is denominated in units of consumption goods. $e_{s}$ denotes household income that is sheltered from taxation. Households have quasilinear preferences with respect to consumption and a convex utility cost with respect to hours worked. Household preferences can be summarized as follows:

$$
\underbrace{\alpha V\left(g_{s}\right)}_{\text {Value of public good }}+\underbrace{\left(1-t_{s}\right) \omega h_{s}}_{\text {After-tax wage income }}+\underbrace{t_{s} e_{s}}_{\text {Tax evasion }}-\underbrace{e_{s} \tau_{s}}_{\text {Cost of tax evasion }}+\underbrace{r_{s}^{J}}_{\text {Gov transfers }}-\underbrace{\frac{h_{s}^{1+\frac{1}{\varepsilon}}}{1+\varepsilon}}_{\text {Labour disutility }}
$$

Households choose tax evasion $e_{s}$ and work effort $h_{s}$ to maximize:

$$
\max _{h_{s}, e_{s} \in\left[0, \omega h_{s}\right]}\left(1-t_{s}\right) \omega h_{s}+t_{s} e_{s}-e_{s} \tau_{s}-\frac{h_{s}^{1+\frac{1}{\varepsilon}}}{1+\varepsilon} .
$$

The choice of tax evasion is then simply

$$
e_{s}=\epsilon\left(t_{s}, \tau_{s}\right) \equiv\left\{\begin{array}{cc}
0 & t_{s} \leq \tau_{s} \\
\omega h_{s} & t_{s}>\tau_{s}
\end{array}\right.
$$

Households conceal their entire income if the cost of evasion is lower than the tax rate, and pay their tax liabilities in full otherwise. We assume that households comply when they are indifferent between evasion and payment. The choice of labour supply is

$$
h_{s}=H\left(t_{s}\right) \equiv\left[\varepsilon\left(1-t_{s}\right) \omega\right]^{\varepsilon} .
$$

Household indirect utility is therefore

$$
u\left(g_{s}, t_{s}, \tau_{s}, r_{s}^{J}\right)=\alpha V\left(g_{s}\right)+\frac{\varepsilon^{\varepsilon}\left(\left(1-t_{s}\right) \omega\right)^{\varepsilon+1}}{\varepsilon+1}+\left(t_{s}-\tau_{s}\right) \epsilon\left(t_{s}, \tau_{s}\right)+r_{s}^{J} .
$$


In contrast to the benchmark model, the supply of labour is elastic and taxes are distortionary. Investments in fiscal capacity $\tau_{s}$ increases tax efficiency, defined as $\frac{\partial \tilde{R}\left(t_{s}, \tau_{s}\right)}{\partial t_{s}}$, where $\tilde{R}\left(t_{s}, \tau_{s}\right)$ are tax revenues given by

$$
\tilde{R}\left(t_{s}, \tau_{s}\right)=t_{s}\left(\omega h_{s}-e_{s}\right)=\varepsilon^{\varepsilon} \omega^{\varepsilon+1} t_{s}\left(1-t_{s}\right)^{\varepsilon}-t_{s} \epsilon\left(t_{s}, \tau_{s}\right) .
$$

Given the evasion function (1), tax efficiency is then

$$
\frac{\partial \tilde{R}\left(t_{s}, \tau_{s}\right)}{\partial t_{s}}=\left\{\begin{array}{c}
\varepsilon^{\varepsilon}\left(1-t_{s}\right)^{\varepsilon} \frac{1-t_{s}-\varepsilon t_{s}}{1-t_{s}} \\
0 \quad t_{s}>\tau_{s}
\end{array} \quad t_{s} \leq \tau_{s}\right.
$$

Fiscal capacity $\tau_{s}$ increase tax efficiency because higher fiscal capacity increases the range of values $t_{s}$ for which tax efficiency is strictly positive.

A more general formulation, where tax efficiency is monotonically increasing in $\tau_{s}$ is possible. In such a formulation, the cost of tax evasion could take a more general form, for example $C\left(e_{s}, \tau_{s}\right)$, giving a differentiable function $\epsilon\left(t_{s}, \tau_{s}\right)$, which in turn would lead to a tax efficiency function that is increasing in $\tau_{s}$ for all $t_{s}$. The more general formulation would complicate analysis significantly with no obvious gains in insight.

\subsubsection{Choice of policy}

In each period $s$, the incumbent collects tax revenues of $\tilde{R}\left(t_{s}, \tau_{s}\right)$ and uses these resources to (a) provide public goods $g_{s}$, (b) accumulate fiscal (tax enforcement) capacity at cost $c\left(\tau_{s+1}-(1-d) \tau_{s}\right)$ and (c) make transfers $\left\{r_{s}^{J}\right\}_{J=I, O}$. Given $\tau_{s}$, a policy $\left\{t_{s}, g_{s}, \tau_{s+1}\right\}$ gives group $J$ a period $s$ indirect utility of

$W\left(t_{s}, \tau_{s+1}, g_{s} ; \tau_{s}, \beta^{J}\right) \equiv \begin{gathered}\alpha V\left(g_{s}\right)+\beta^{J}\left[\tilde{R}\left(t_{s}, \tau_{s}\right)-g_{s}-c\left(\tau_{s+1}-(1-d) \tau_{s}\right)\right] \\ +\left(t_{s}-\tau_{s}\right) \epsilon\left(t_{s}, \tau_{s}\right)+\frac{\varepsilon^{\varepsilon}\left(\left(1-t_{s}\right) \omega\right)^{\varepsilon+1}}{\varepsilon+1}\end{gathered}$,

where $J \in\{I, O\}, \beta^{I}=2(1-\theta)$ and $\beta^{J}=2 \theta$.

The incumbent's choice of $t_{s}$ is static and can be described as follows. For any choice $t_{s}>\tau_{s}, \epsilon\left(t_{s}, \tau_{s}\right)=\omega h_{s}$ and $\tilde{R}\left(t_{s}, \tau_{s}\right)=0$. Taxes do not raise revenues and no such choice can be optimal for the incumbent. The problem can be reframed by setting $\epsilon\left(t_{s}, \tau_{s}\right)=0$ and with the additional constraint 
$t_{s} \leq \tau_{s}$. Indirect utility can then be reformulated as

$$
W\left(t_{s}, \tau_{s+1}, g_{s} ; \tau_{s}, \beta^{J}\right) \equiv \begin{gathered}
\alpha V\left(g_{s}\right)+\beta^{J}\left[R\left(t_{s}\right)-g_{s}-c\left(\tau_{s+1}-(1-d) \tau_{s}\right)\right] \\
+\frac{\varepsilon^{\varepsilon}\left(\left(1-t_{s}\right) \omega\right)^{\varepsilon+1}}{\varepsilon+1}
\end{gathered}
$$

where

$$
R\left(t_{s}\right) \equiv \varepsilon^{\varepsilon} \omega^{\varepsilon+1} t_{s}\left(1-t_{s}\right)^{\varepsilon}
$$

The incumbent's maximization problem is then

$$
\begin{gathered}
U^{I}(\tau) \equiv \max _{t, \tau^{\prime}, g}\left\{W\left(t, \tau^{\prime}, g ; \tau, 2(1-\theta)\right)+\delta Z^{I}\left(\tau^{\prime}\right)\right\} \\
\text { s.t. } \quad R(t)-g-c\left(\tau^{\prime}-(1-d) \tau\right) \geq 0 \\
t \leq \tau,
\end{gathered}
$$

with $U^{I}(\tau)$ the incumbent's value function, $Z^{I}(\tau) \equiv(1-\gamma) U^{I}(\tau)+\gamma U^{O}(\tau)$ his continuation value, time subscripts suppressed, and $\tau^{\prime}$ denoting $\tau_{s+1}$.

The static first order condition

$$
\alpha V_{g}(g)=\lambda+2(1-\theta),
$$

is now combined with

$$
\alpha V_{g}(g)=\zeta+\mu(t)
$$

where $\lambda$ is the Lagrange multiplier on (3) and

$$
\mu(t) \equiv \frac{1-t}{1-t(\varepsilon+1)}
$$

is the marginal cost of public funds. $\zeta>0$ only if $t=\tau$, i.e. if tax capacity constrains the incumbent's choice of the tax rate.

The first of these first order conditions states that the marginal value of the public good must equal its marginal cost. The second of these equations states that the marginal value of the public good equals the marginal cost of public funds, unless taxation is limited by institutional rather than economic constraints.

Finally we have

$$
\lim _{t \backslash \tau^{\prime}} \delta Z_{\tau}^{I}(t) \leq c \alpha V_{g}(g) \leq \lim _{t \nearrow \tau^{\prime}} \delta Z_{\tau}^{I}(t),
$$


which degenerates to

$$
c \alpha V_{g}(g)=\delta Z_{\tau}^{I}\left(\tau^{\prime}\right),
$$

if $Z$ (.) is differentiable at the chosen value of $\tau^{\prime}$.

\subsection{Equilibrium}

Our equilibrium concept is as in the benchmark model: A Symmetric Markov Perfect Equilibrium (MPE). Here too, we restrict attention to equilibria that are limits to a finite horizon economy.

\subsubsection{Characterizing the equilibrium}

There exists a level of fiscal capacity $\tilde{\tau}$, above which redistribution occurs. Thus $\tilde{\tau}$ partitions the state space into a common-interest regime, where all tax revenues are allocated to public goods including fiscal capacity, and a redistributive regime, where redistribution occurs and $g=\hat{g}$, defined implicitly by

$$
\alpha V(\hat{g})=2(1-\theta) .
$$

There are now two cases, depending on whether economic constraints (tax distortions) or institutional constraints (fiscal capacity) limit taxation. If fiscal capacity is sufficiently high so that the tax rate can be chosen freely, $t_{s}$ is chosen jointly with public spending so as to equalize the marginal cost of taxation with the marginal value of public goods. This is done according to (4) with $\zeta=0$.

In the common-interest regime, policy is set to $\left\{t^{*}(\tau), g^{*}(\tau), T^{*}(\tau)\right\}$, which jointly solve

$$
\alpha V_{g}\left(g^{*}(\tau)\right)=\mu\left(t^{*}(\tau)\right),
$$

(6) and (3) holding with equality. $g^{*}(\tau)$ and $T^{*}(\tau)$ are increasing in $\tau$, while $t^{*}(\tau)$ is decreasing in $\tau$.

In the redistributive regime, $g=\hat{g}$, and (4) gives $t=\hat{t}$, defined implicitly by

$$
\mu(\hat{t})=2(1-\theta) \cdot 1
$$

${ }^{1}$ This can be solved to give a closed form solution for $\hat{t}$ :

$$
\hat{t} \equiv \frac{1-2 \theta}{1-2 \theta+2(1-\theta) \varepsilon}
$$


$\hat{t}$ gives the highest tax rate that a redistributing incumbent would be willing to bear to finance redistribution, due to the ensuing tax distortions. $\hat{t}$ is decreasing in $\theta$, as the value of redistribution is lower in a more cohesive society, justifying lower taxes to finance such redistribution. $\hat{t}$ is also decreasing in $\varepsilon$ as taxes are less distortionary the lower is the elasticity of labour supply.

As $t^{*}(\tau)$ is strictly decreasing in $\tau$, there is thus a threshold value $\tau^{*}$, below which $\tau<t^{*}(\tau)$ and fiscal capacity constrains taxation and above which $\tau>t^{*}(\tau)$ and tax distortions constrain taxation.

We can summarize the equilibrium as follows. If $\tau>\tau^{*}$, taxes are set at $\left\{t^{*}(\tau), g^{*}(\tau), T^{*}(\tau)\right\}$. If $\tau<\tau^{*}$ then $t=\tau$ and $\left\{g, \tau^{\prime}\right\}$ solve (6) and the budget constraint (3) holding with equality if $g<\hat{g}$ or $g=\hat{g}$ otherwise.

In the benchmark model, the state space was partitioned by a value $\tilde{\tau}$, above which the economy operated in the redistributive regime, and under which it operated in the common-interest regime. In this appendix, the state space is additionally partitioned by the value $\tau^{*}$, above which taxation is constrained by tax distortions, and below which it is constrained by fiscal capacity.

\subsection{Pigouvian Planner}

Consider first the problem of a Pigouvian planner who is in power indefinitely and puts equal weights on the welfare of all citizens. This corresponds to the case $\theta=\frac{1}{2}$ and $\gamma=0$. In this case $Z^{I}(\tau)=U^{I}(\tau)$ and the problem becomes a standard dynamic programming problem, where $U^{I}(\tau)$ is a concave function and

$$
U_{\tau}^{I}(\tau)=\left\{\begin{array}{c}
\left(R_{t}(\tau)+(1-d) c\right) \alpha V_{g}(g)-\varepsilon^{\varepsilon} \omega^{\varepsilon+1}(1-\tau)^{\varepsilon} \quad \text { if } \tau<\tau^{*}, \\
(1-d) c \alpha V_{g}(g) \quad \text { if } \tau>\tau^{*}
\end{array}\right.
$$

where $\tau^{*}$ is defined in the previous section. Noting that

$$
R_{t}(t)=\frac{\omega H(t)}{\mu(t)}
$$


and we can rewrite the marginal value of fiscal capacity as

$$
U_{\tau}^{I}(\tau)=\left\{\begin{array}{c}
\left(\frac{\omega H(\tau)}{\mu(\tau)}+(1-d) c\right) \alpha V_{g}(g)-\omega H(\tau) \quad \text { if } \tau<\tau^{*} \\
(1-d) c \alpha V_{g}(g) \quad \text { if } \tau>\tau^{*}
\end{array} .\right.
$$

As $\mu(\tau)=\alpha V_{g}(g) \forall \tau>\tau^{*}, U_{\tau}^{I}(\tau)$ is continuous at $\tau=\tau^{*}$.

With $Z^{I}(\tau)=U^{I}(\tau)$, (6) reads

$$
c \alpha V_{g}(g)=\delta U_{\tau}^{I}\left(\tau^{\prime}\right)
$$

and for a choice $\tau^{\prime}>\tau^{*}(9)$ then gives

$$
V_{g}(g)=\delta(1-d) V_{g}\left(g^{\prime}\right)
$$

As $\delta(1-d)<1$, this implies a declining path of fiscal capacity and there can be no steady state with $\tau>\tau^{*}$. If there is "excess" fiscal capacity, in the sense that tax distortions rather than fiscal capacity are the binding constraint on taxation, the incumbent will decummulate fiscal capacity until institutional-rather than economic-constraints limit taxation.

Now consider a choice $\tau^{\prime}<\tau^{*}$. We obtain the Euler equation

$$
c \alpha V_{g}(g)=\delta\left(\frac{\omega H(\tau)}{\mu(\tau)}+(1-d) c\right) \alpha V_{g}(g)-\delta \omega H(\tau) .
$$

This system has a unique steady state at

$$
\alpha V_{g}\left(R\left(\tau^{P}\right)+c d \tau^{P}\right)=\mu\left(\tau^{P}\right) \frac{\omega H\left(\tau^{P}\right)}{\omega H\left(\tau^{P}\right)+c\left(1-d-\frac{1}{\delta}\right) \mu\left(\tau^{P}\right)} .
$$

Recalling (4), $\tau^{P}<\tau^{*}$ because

$$
\frac{\omega H\left(\tau^{P}\right)}{\omega H\left(\tau^{P}\right)+c\left(1-d-\frac{1}{\delta}\right) \mu\left(\tau^{P}\right)}>1
$$

and therefore $\zeta>0$ at the steady state $\tau^{P}$.

This points to a qualitative contrast between our model and that of Battaglilni and Coate (2007, 2008). In Battaglini and Coate (2007, 2008) the marginal value of the public good is always equal to the marginal cost of public funds and economic constraints limit taxation. Here, the marginal 
value of the public good exceeds the marginal cost of public funds $\mu(t)$ in steady state.

In a Pigouvian steady state, the marginal benefit of public good provision is set equal to the marginal cost of maintaining the steady state level of fiscal capacity. This cost comprises both the distortionary cost of taxation and the cost of fiscal capacity maintenance. Thus the marginal value of public goods strictly exceeds the marginal cost of public funds, and the distortionary cost of taxation is not the binding constraint on the chosen level of taxation.

\subsection{Political Economy}

We now outline the conditions for steady states in the three possible regions: common interest, redistributive, and a "weak" state at the boundary between the common interest and redistributive regimes. As in the benchmark model, two critical conditions determine the existence of each type of steady state. Due to the additional complication of distortionary taxation, however, we are unable to obtain closed-form solutions for these conditions.

The cohesiveness condition determines the existence of a common-interest steady state. As in the benchmark model, a common-interest steady state is attainable only if the Pigouvian steady state analyzed in the previous section is within the common-interest regime. This yields the following condition:

The Cohesiveness Condition: $R\left(\tau^{P}\right)-c d \tau^{P}<\hat{g}$

The definition of $\tau^{P}$ in (10) is not a function of the political-economy parameters $\gamma$ and $\theta$. Thus the cohesiveness condition is a condition on $\theta$ alone (via $\hat{g}$ ) and holds for values of $\theta$ that are sufficiently close to $\frac{1}{2}$.

The second condition is the stability condition. When the stability condition holds, a redistributive steady state exists.

The Stability Condition: $R\left(\tau^{R}\right)-c d \tau^{R}>\hat{g}$,

where $\tau^{R}=\min \{\hat{t}, \hat{\tau}\}$ and $\hat{\tau}$ is defined implicitly via

$$
2(1-\theta) c=2 \phi \delta\left[\frac{\omega H(\hat{\tau})}{\mu(\hat{\tau})}+c(1-d)\right]-\delta \omega H(\hat{\tau}) .
$$

As in the benchmark model, the stability condition is a function of $\theta$ and $\gamma$. $\phi \equiv(1-\gamma)(1-\theta)+\gamma \theta$ is an important stability parameter. It determines 
the expected share of next period's transfers obtained by the incumbent. As $\hat{\tau}$ is increasing in $\phi$ in (11), the stability condition is more likely to hold the larger is $\phi$, as in the benchmark model.

We now explore the relationship between the cohesiveness and stability conditions in the benchmark model and the ones presented here. An inelastic labour supply is equivalent to $\varepsilon \rightarrow 0$. As $\varepsilon \rightarrow 0, \mu(\tau) \rightarrow 1$ and $H(t) \rightarrow \omega$. Then (10) gives

$$
\alpha V_{g}\left(g^{P}\right)=\frac{\omega}{\omega+c\left(1-d-\frac{1}{\delta}\right)},
$$

and the cohesiveness condition becomes

$$
\frac{\omega}{\omega+c\left(1-d-\frac{1}{\delta}\right)}>2(1-\theta)
$$

which is precisely the cohesiveness condition in the body of the paper.

When labour supply is inelastic, (11) is no longer a function of $\tau$, as its left hand side remains $2(1-\theta) c$, but its right hand side becomes $2 \phi \delta(\omega+c(1-d))-$ $\delta \omega$. These are precisely the marginal cost and marginal benefit, respectively, of remaining in a redistributive steady state indefinitely in the benchmark model. If the cost exceeds the benefit then no redistributive steady state exists, as an incumbent in the redistributive regime would choose to move to the common interest regime in the following period. If the benefit strictly exceeds the cost-as is the case when the stability condition holds in the benchmark model-an incumbent in the redistributive regime wishes to accumulate fiscal capacity with no bound, and a redistributive steady state exists at $\tau^{R}=\bar{\tau}$.

Put differently, in the benchmark model, $\tau^{R}$ is always at a corner solution. If the benchmark stability condition holds, $\tau^{R}=\bar{\tau}$, and if it fails to hold $\tau^{R}=0$. In the benchmark model, $\bar{\tau}$ was assumed to be such that $\alpha V_{g}(R(\bar{\tau})-c d \bar{\tau})<2(1-\theta)$, so that that our new stability condition holds whenever the stability condition in the benchmark model holds. If the benchmark stability condition does not hold then $\tau^{R}=0$ and the stability condition here cannot either.

A qualitative difference between this model and the benchmark model emerges due to the difference between the stability conditions of these two models. The candidate redistributive steady state in the benchmark model is the corner-solution $\bar{\tau}$, which is always in the redistributive regime. Here, due to the convexity created by distortionary taxation, an interior candidate 
for a redistributive steady state $\tau^{R}$ emerges. An additional condition must be satisfied for a redistributive steady state to exist. The value of $\tau^{R}$ must be in the redistributive regime, which corresponds to the stability condition listed above.

Unlike the benchmark model, the cohesiveness and stability conditions are not mutually exclusive. In the benchmark model, the candidate redistributive steady state is zero whenever the cohesiveness condition holds. Thus $\tau^{R}<\tau^{P}$ whenever the cohesiveness condition holds, and the overlap between the cohesiveness and stability conditions is degenerate. Here, however, $\tau^{R}$ is endogenous and interior. Its value may thus exceed or be lower than $\tau^{P}$, allowing for the stability condition, the cohesiveness condition, both, or neither, to hold.

We also note that the marginal value of the public good $\alpha$, which is central to equilibrium determination in Battaglini and Coate (2007) reemerges as a key parameter in our model with distortionary taxation. High values of $\alpha$ increase $\hat{g}$, which now appears in both the stability and the cohesiveness conditions. Higher levels of $\alpha$ make it more likely for the cohesiveness condition to hold and less likely that stability condition holds.

\subsection{Discussion}

In the following section we formally prove the main claim of this appendix. Modified cohesiveness and stability conditions partition the state space into regions, each corresponding to one of the three steady states in the benchmark model. In this section, we show that the model with distortionary taxation yields very similar results to those in the benchmark model.

We cannot obtain analytical solutions for the cohesiveness and stability conditions when taxes are distortionary. We resort to computational methods. $^{2}$ The following figures show the values of $\gamma$ and $\theta$ required for the existence of each type of steady state. We plot these figures for different values of $\alpha$, which is now an important parameter in determining the type of steady state that emerges.

\footnotetext{
${ }^{2}$ For the sake of comparison, we use the same parameter values as were used to plot Figure 7 in the benchmark model. These parameters were chosen for graphical convenience rather than an attempt to "calibrate" to empirical values: $c=2, \omega=0.9, d=0.17$ and $\delta=0.95$. Two additional parameters are introduced in the general model. We set $\varepsilon=2$, an elasticity commonly used in the literature, and vary $\alpha$ to demonstrate its effects.
} 


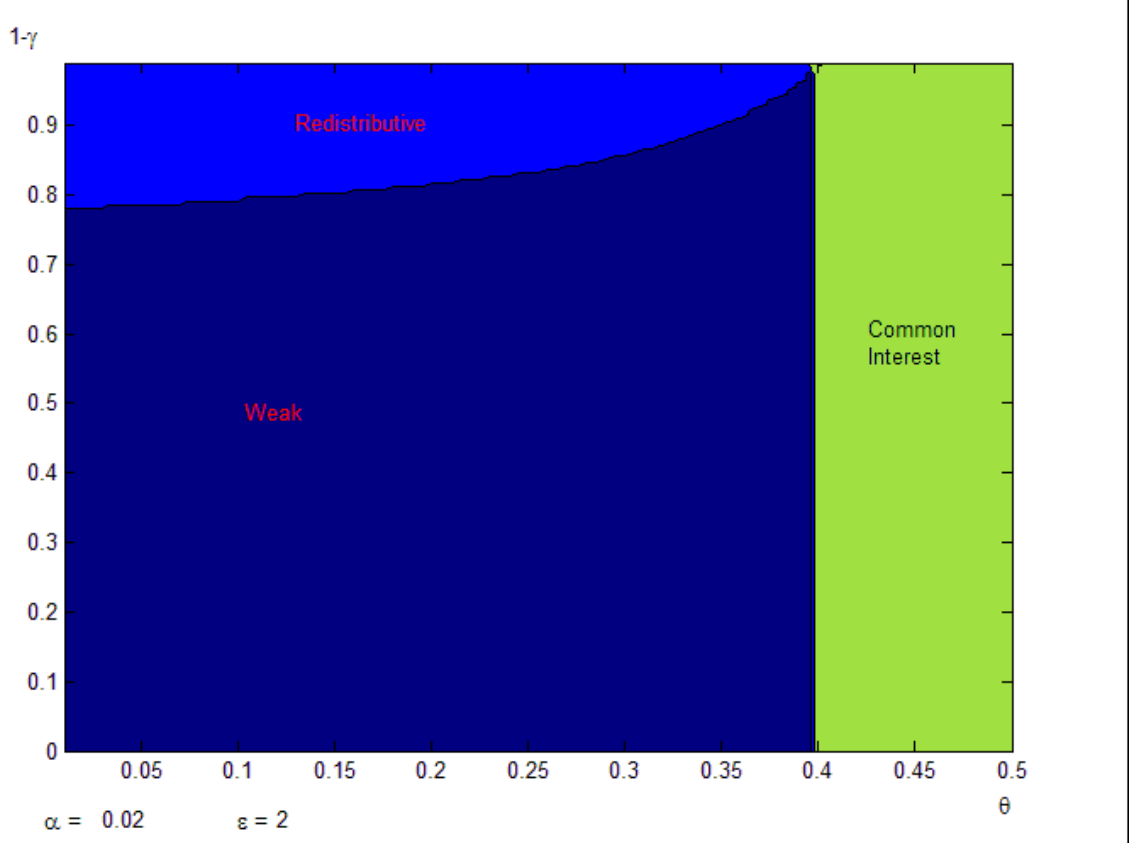

Figure 1: Three types of steady state $(\alpha=0.02)$

The first of these figures is plotted for a value of $\alpha$ that gives an almost identical picture to that in benchmark model. This demonstrates that the result in the benchmark model is largely robust to the introduction of distortionary taxation.

The second figure shows the effects of an increase in $\alpha$. A higher level of $\alpha$ increases the range where the common interest state exists and decreases the range where the redistributive state exists. The latter parameter values also allow for a region where both the stability and cohesiveness conditions hold. In the region of overlap, a redistributive steady state exists.

In contrast to Battaglini and Coate (2007) our equilibrium refinement method ensures a unique equilibrium. A finite horizon economy has a unique equilibrium, as does its limit as the time horizon $S$ grows without a bound. As will be shown in the following section, when the stability and cohesiveness conditions both hold, a redistributive steady state results.

When the stability condition holds, the belief that future incumbents will follow redistributive policies encourages incumbents to over-accumulate fiscal capacity for the purpose of redistribution. A redistributive steady state 


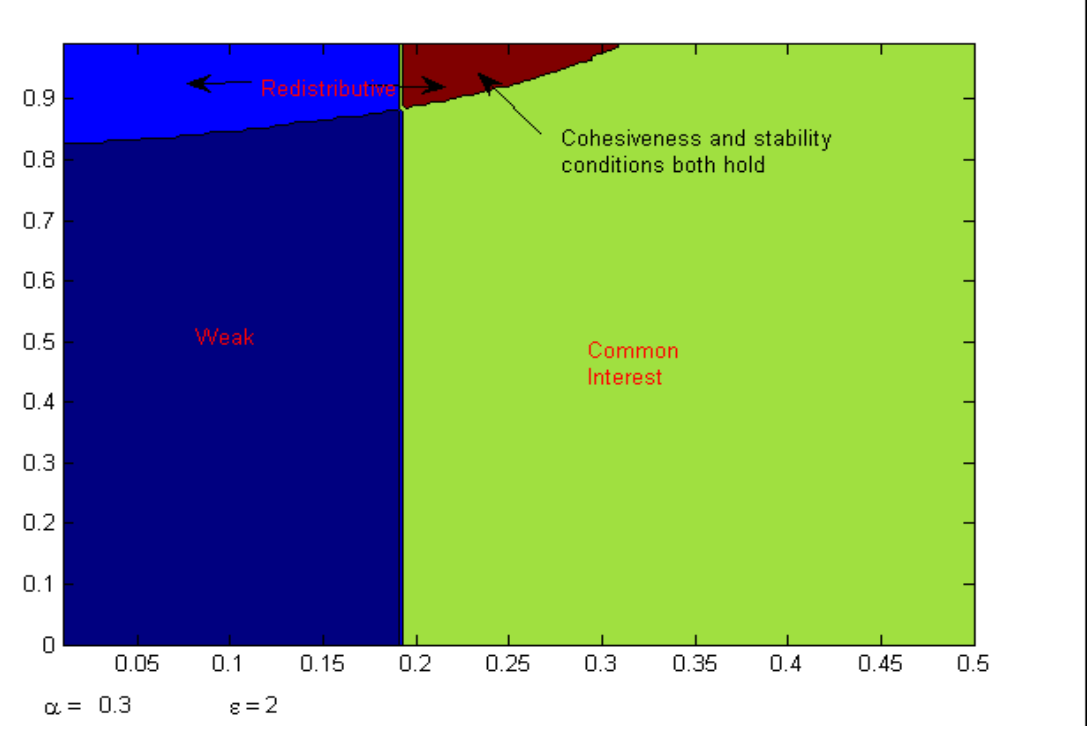

Figure 2: Three types of steady state $(\alpha=0.3)$

then emerges. When the cohesiveness condition holds, the belief that future incumbents will behave "responsibly" and remain at the Pigouvian steady state dissuades incumbents from accumulating excessive fiscal capacity.

Multiple equilibria may exist in Battaglini and Coate (2007) for some parameter values because either type of steady state can be supported by the belief that future policy makers will follow a similar policy rule.

In a finite-horizon economy, the period $S$ incumbent cannot commit to anything but scrapping fiscal capacity for the purpose of redistribution, if he inherits a sufficiently large level of fiscal capacity. The period $S-1$ incumbent, knowing this, follows a "redistributive" policy rule, knowing that his successor will do the same. Previous incumbents follow suit. The commoninterest equilibrium is thus eliminated.

\subsection{Proofs}

In this section, we prove the claims of section 1.4. A redistributive steady state exists when the stability condition holds. When the stability condition does not hold but the cohesiveness condition holds, a common-interest steady 
state exists. A weak steady state-at the boundaries of the two regimesemerges when neither condition holds.

We consider an economy of finite horizon $S$, and analyze its limit as $S \rightarrow \infty$. In each period $s$, we can solve for continuation values $U^{I, s}\left(\tau_{s}\right)$, $U^{O, s}\left(\tau_{s}\right), Z^{I, s}\left(\tau_{s}\right)$, and policy functions $G^{s}\left(\tau_{s}\right)$ and $T^{s}\left(\tau_{s}\right)$. The policy functions will imply a choice of a tax rate $t_{s}$.

\subsubsection{Period S}

In period $S, T^{S}(\tau)=0$ trivially, as future fiscal capacity has no value. There thus exists a value $\tilde{\tau}_{S}$, such that $\forall \tau_{S} \geq \tilde{\tau}_{S}, g=\hat{g}$. In addition, as in section 1.2 , there is a value $\tau_{S}^{*}$ below which $t_{S}=\tau_{S}$ and above which $t_{S}=t^{*}\left(\tau_{S}\right)$.

For any $\tau_{S} \leq \tilde{\tau}_{S}$, we are in the common interest regime, and all tax revenues are used as public goods. $g_{S}=G^{S}\left(\tau_{S}\right)$ which solves

$$
G^{S}\left(\tau_{S}\right)=R\left(t_{S}\right)+c(1-d) \tau_{S}
$$

where $t_{S}=\min \left\{\tau_{S}, t^{*}\left(\tau_{S}\right)\right\} . G\left(\tau_{S}\right)$ is strictly increasing $\forall \tau_{S}<\tilde{\tau}_{S}$.

Characterizing $\tilde{\tau}_{S}$ The threshold for redistribution depends on whether $\tilde{\tau}_{S}$ is greater than or smaller than $\tau_{S}^{*}$. If $\tilde{\tau}_{S}>\tau_{S}^{*}$ then fiscal capacity is not a constraint to taxation when in the redistributive regime and $t_{S}=\hat{t} \forall \tau_{S} \geq \tilde{\tau}_{S}$. $\tilde{\tau}_{S}$ then solves

$$
\tilde{\tau}_{S}=\frac{\hat{g}-R(\hat{t})}{c(1-d)} .
$$

Otherwise, $\tilde{\tau}_{S}<\tau_{S}^{*}=\hat{t}$ and fiscal capacity constrains taxation to $t_{S}=\tilde{\tau}_{S}$. $\tilde{\tau}_{S}$ then solves

$$
R\left(\tilde{\tau}_{S}\right)+c(1-d) \tilde{\tau}_{S}=\hat{g}
$$

$\tilde{\tau}_{S}<\tau_{S}^{*}$ if and only if

$$
R(\hat{t})+c(1-d) \hat{t}>\hat{g}
$$

which is a condition on parameter values alone.

Period S value functions Given the period $S$ policy functions outlined above, we can characterize $Z^{I, S}\left(\tau_{S}\right)$. This can be done concisely by noting 
that

$$
Z_{\tau}^{I, S}\left(\tau_{S}\right)=\left\{\begin{array}{c}
\psi\left(\tau_{S}\right)\left[\frac{\omega H\left(\tau_{S}\right)}{\mu\left(\tau_{S}\right)}+c(1-d)\right]-\omega H\left(\tau_{S}\right) \quad \text { for } \tau_{S}<\tau_{S}^{*} \\
\psi\left(\tau_{S}\right) c(1-d) \quad \text { for } \tau_{S}>\tau_{S}^{*}
\end{array}\right.
$$

where $\psi\left(\tau_{s}\right)=2 \phi$ when $\tau_{s}>\tilde{\tau}_{s}$ and $\psi\left(\tau_{s}\right)=\alpha V_{g}\left(G\left(\tau_{s}\right)\right)$ when $\tau_{s}<\tilde{\tau}_{s}$. We thus have a strictly concave value function wherever $\tau_{S}<\tau_{S}^{*}$, wherever fiscal capacity is the binding constraint on taxation.

Choice of $\tau_{S}$ in period S-1 Now consider the choice of $\tau_{S}$ in period $S-1$. This choice is governed by (6). We first point out that a choice $\tau_{S}>\max \left\{\tau_{S}^{*}, \tilde{\tau}_{S}\right\}$ cannot be optimal, as (6) would then state

$$
c \alpha V_{g}\left(g_{S-1}\right)=2 \phi \delta c(1-d)
$$

But as $c \alpha V_{g}\left(g_{S-1}\right)>2(1-\theta)>2 \phi \delta c(1-d)$, this first order condition cannot hold.

We now provide a condition for a choice $\tau_{S}>\tilde{\tau}_{S}$, i.e. in the redistributive regime. It is summarized in the following lemma.

Lemma 1 If the incumbent in period $S-1$ is in the redistributive regime, he will choose to put the economy in the redistributive regime in period $S$ if and only if

$$
R\left(\tau^{R}\right)+c(1-d) \tau^{R}>\hat{g}
$$

where $\tau^{R}=\min \{\hat{\tau}, \hat{t}\}$, with $\hat{\tau}$ defined as in (11).

Proof. Throughout the proof of this lemma, we will consider the case where the period $S-1$ incumbent is in the redistributive regime, so that $\alpha c V_{g}\left(g_{S-1}\right)=2(1-\theta) c$. To prove this lemma, we return to the two cases discussed in section 1.6.1. First, assume that (13) does not hold so that $\tilde{\tau}_{S}>\tau_{S}^{*}$. Then $\tau_{S}>\tilde{\tau}_{S}$ implies $\tau_{S}>\max \left\{\tau_{S}^{*}, \tilde{\tau}_{S}\right\}$, and a choice of $\tau_{S}$ in the redistributive regime cannot be optimal. (13) is therefore a necessary condition for the period $S-1$ incumbent to choose $\tau_{S}>\tilde{\tau}_{S}$.

If, on the other hand, (13) is satisfied and $\tilde{\tau}_{S}<\hat{t}$, then the unique choice of $\tau_{S}$-if chosen in the redistributive regime-is $\tau_{S}=\tau^{R}$, according to (6) and (14). An additional necessary condition for $\tau_{S}$ to be in the redistributive regime is that $\tau^{R}$ is in the redistributive regime. $\tau^{R}>\tilde{\tau}_{S}$ is equivalent to (15) 
Together (13) and (15) are necessary and sufficient conditions for redistribution in period $S$, but as (13) holds only if (15) does, we are left with the condition (15) as a necessary and sufficient condition, as claimed.

The discussion of the terminal period and the choice of fiscal capacity available in the terminal period did not depend on whether the stability and cohesiveness conditions held. We now discuss separately the case where the stability condition holds and where it does not hold.

\subsubsection{Redistributive Steady State}

We now consider parameters where the stability condition holds and show that a redistributive steady state exists. If the stability condition holds, (15) must hold as well. According to Lemma 1, if the period $S-1$ incumbent is in the redistributive regime, he will chose $\tau_{S}=\tau^{R}$. There then exists a value $\tau_{S-1}=\tilde{\tau}$ above which $\left\{g_{S}, \tau_{S}\right\}=\left\{\hat{g}, \tau^{R}\right\}$ will be chosen.

Characterizing $\tilde{\tau} \quad \tilde{\tau}$ is the lowest value of $\tau_{S-1}$, for which $\left\{\tau^{R}, \hat{g}\right\}$ are feasible. If $\tilde{\tau}>\hat{t}$, then

$$
\tilde{\tau}=\frac{\hat{g}+c \tau^{R}-R(\hat{t})}{c(1-d)}
$$

If $\tilde{\tau}<\hat{t}$ then $\tilde{\tau}$ solves

$$
R\left(\tilde{\tau}_{S-1}\right)+c(1-d) \tilde{\tau}_{S-1}-c \tau_{S}^{R}=\hat{g} .
$$

$\tilde{\tau}<\hat{t}$ if and only if

$$
R(\hat{t})+c(1-d) \hat{t}-c \tau^{R}>\hat{g}
$$

Choice of $\tau_{S-1}$ We can now characterize the period $S-1$ continuation value in the redistributive regime:

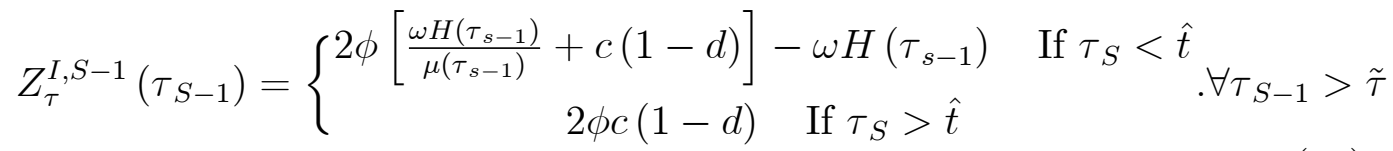

The choice of $\tau_{S-1}$ is governed by (6). As with $\tau_{S}$, a choice $\tau_{S-1}>\max \{\tilde{\tau}, \hat{t}\}$ cannot be optimal, as then (6) would require $2(1-\theta) c=2 \phi c(1-d)$. We can therefore show that 
Lemma 2 If the incumbent in period $S-2$ is in the redistributive regime, he will choose to put the economy in the redistributive regime in period $S-1$ if and only if the stability condition holds.

Proof. The proof of this lemma is almost identical to that of Lemma 2. If $\tau_{S-1}$ is chosen in the redistributive regime, $\tau_{S-1}=\tau^{R}$ according to (6) and (16). $\tau^{R}$ is in fact in the redistributive regime $\left(\tau^{R}>\tilde{\tau}\right)$ if and only if the stability condition holds.

Redistributive steady state The analysis for period $S-2$ holds identically for periods $s<S-2$. If the economy is in the redistributive regime in period $s$, it will remain in the redistributive regime in period $s+1$ if and only if the stability condition holds. In addition $\tau_{s+1}=\tau^{R}$ is the chosen level of fiscal capacity in the redistributive regime. Thus as $S \rightarrow \infty$ this economy has a steady state at $\tau=\tau^{R}$ if and only if the stability condition holds. To summarize

Proposition 3 A redistributive steady state at $\tau=\tau^{R}$ exists if and only if the stability condition holds.

\subsubsection{When the stability condition does not hold}

Having seen that the stability condition is a necessary and sufficient for the existence of a redistributive steady state at $\tau=\tau^{R}$, we now consider parameter values such that the stability condition fails to hold.

As indicated in Lemma 2, the stability condition determines whether the economy will be in the redistributive regime, in any period other than $S$. The weaker condition (15) determines whether the economy will arrive at period $S$ with sufficient fiscal capacity to be in the redistributive regime. Analysis is simplified by assuming that not only the stability condition, but also (15), fails to hold. We show that as $S \rightarrow \infty$, the economy is identical to that of the Pigouvian planner for all $\tau<\tilde{\tau}$. Following this analysis, we will relax this assumption and show that even if (15) holds, so that redistribution may occur in period $S$, the limiting economy as $S \rightarrow \infty$ is nevertheless the same.

Lemma 1 implies that if (15) does not hold, the economy will not be in the redistributive regime in period $S$. Similarly, if the stability condition fails to hold, the economy will not be in the redistributive regime along the equilibrium path for any period $s>0$, according to Lemma 2. Therefore, 
for any $s>0$, the continuation values of the incumbent and opposition are the same and $U^{I, s}\left(\tau_{s}\right)=U^{O, s}\left(\tau_{s}\right)=Z^{I, s}\left(\tau_{s}\right)$. As $S \rightarrow \infty$ this economy becomes identical to that of the Pigouvian planner analyzed in Section 1.3 for all $\tau<\tilde{\tau}$.

We then have two cases, depending on whether $\tilde{\tau}$ is greater than or smaller than the candidate steady state $\tau^{P}$. If $\tilde{\tau}>\tau^{P}$, the Pigouvian steady state is attainable along the equilibrium path and is the unique steady state. The condition $\tilde{\tau}>\tau^{P}$ is given by the cohesiveness condition, so that we may conclude:

Proposition 4 If the stability condition does not hold, but the cohesiveness condition holds, the unique steady state $\tau^{C}$ is in the common interest regime and is at $\tau^{C}=\tau^{P}$.

If the cohesiveness condition does not hold, $\tilde{\tau}<\tau^{P}$, and a steady state in the common interest regime does not exist. A steady state cannot exist in the redistributive regime either, as the stability condition does not hold. The remaining candidate for a steady state is at $\tau=\tilde{\tau}$.

If $\tilde{\tau}$ is a steady state, (5) must hold at $\tilde{\tau}$. For $\tau<\tilde{\tau}$, the economy is in the common interest regime, where the problem is identical to that of the Pigouvian planner. We thus have

$\lim _{\tau} \nearrow_{\tilde{\tau}} \delta Z_{\tau}^{I}(\stackrel{\circ}{\tau})=\lim _{\tilde{\tau} \nearrow \tilde{\tau}} \delta U_{\tau}^{I}(\stackrel{\circ}{\tau})=2(1-\theta)\left(\frac{\omega H(\tilde{\tau})}{\mu(\tilde{\tau})}+(1-d) c\right)-\omega H(\tilde{\tau})>2(1-\theta) c$

where $\tau^{P}<\tau^{*}$, as shown in section 1.3, ensures that $\tilde{\tau}<\tau^{*}$. The last inequality follows from the fact that the cohesiveness condition fails to hold.

If the economy were to temporarily enter the redistributive regime, it would return to the common-interest regime in the following period, as the stability condition does not hold. Therefore

$$
\lim _{\tilde{\tau} \searrow \tilde{\tau}} \delta Z_{\tau}^{I}(\stackrel{\circ}{\tau})=2 \phi\left[\frac{\omega H(\tilde{\tau})}{\mu(\tilde{\tau})}+c(1-d)\right]-\omega H(\tilde{\tau})<2(1-\theta) c,
$$

where the last inequality holds whenever the stability condition is not satisfied. The conditions for a steady state at $\tau=\tilde{\tau}$ are therefore satisfied. We summarize the result in the following proposition. 
Proposition 5 If neither the stability nor the cohesiveness condition holds, the unique steady state $\tau^{W}$ is at the boundary between the common-interest and redistributive regimes: $\tau^{W}=\tilde{\tau}$.

As noted above, this discussion assumed that (15) did not hold. We now show that relaxing this assumption does not alter any of the conclusions above. If (15) holds in an economy of horizon $S$ then $\forall \tau_{S-1}>\tilde{\tau}_{S-1}, \tau_{S}$ is chosen as $\tau_{S}=\tau^{R}$, as per Lemma 1 . There exist values of $\tau_{S-1}$, for which $\tau_{S}$ will be chosen in the redistributive regime.

Lemma 2, however, still holds and the redistributive regime will not be reached along the equilibrium path for any $s \in(0, S)$. But, the possibility of redistribution in period $S$ nevertheless implies that the value of being in opposition is different than the value of being the incumbent in period $S$. For those values of $\tau_{S-1}$ such that $\tau_{S}>\tilde{\tau}_{S}$ is chosen, the value of being the incumbent and in opposition are different, due to the differential probability of being in power in period $S$. By induction, a similar logic can be applied to any $s<S$.

However, as $S \rightarrow \infty$ the differential reward for being the incumbent and in opposition moves arbitrarily far into the future. Due to discounting, the difference between $U^{I}(\tau)$ and $U^{O}(\tau)$ accordingly approaches zero. With $U^{I}(\tau)=U^{O}(\tau)=Z^{I}(\tau)$, the analysis that led to propositions 4 and 5 holds even when (15) holds.

\section{Other Equilibrium Concepts}

\subsection{Other Markov Equilibria}

We have thus far restricted attention to equilibria emerging from limits of finite horizon economies as the time horizon goes to infinity. This ensured uniqueness of our MPE. This section discusses a different Markov Perfect Equilibria concept wherein functions are assumed to be concave. Unlike Battaglini and Coate $(2007,2008)$ we cannot assume that the value function are strictly concave. Due to the quasilinearity of the objective function, the value function is linear in fiscal capacity in the redistributive regime, leading to a weakly, rather than strictly concave value function. As Battaglini, Palfrey and Nunnari (2012) point out, a large number of equilibria may emerge when the value function is assumed to be weakly concave. 
In an earlier version of the paper we solved the model under the assumption of weakly concave value functions and obtained similar results to those in our benchmark model. When the cohesiveness condition holds, the unique concave equilibrium is identical limiting equilibrium in the current version. The economy's unique steady state is at the Pigouvian planner's steady state. Similarly, when neither the cohesiveness nor the stability conditions hold, the unique steady state is on the boundary between the common-interest and redistributive regimes: the weak state.

When the stability condition holds, however, a multiplicity of equilibria may exist and steady states other than $\tau^{R}=\bar{\tau}$ may emerge. To illustrate this point, we now construct an additional concave equilibrium that could arise when the stability condition holds.

Consider first the decision rule $T(\tau)$ when $\tau>\tilde{\tau}$. One equilibrium is as in our benchmark model, where $T(\tau)=\bar{\tau} \forall \tau>\tilde{\tau}$. At this candidate steady state

$$
\delta Z_{\tau}^{I}(\tau)=2 \phi \delta(\omega+(1-d) c)-\delta \omega
$$

and the first order condition for fiscal capacity accumulation is satisfied by the stability condition.

Another possibility following from the weak concavity of the continuation value, is that $T(\tau)$ gives values within a convex set $\tau \in \Theta$, where all values $\tau \in \Theta$ are themselves within the redistributive regime.

For values $\tau>\tilde{\tau}$,

$U^{J}(\tau)=\alpha V(\hat{g})+\beta^{J}[(\omega+(1-d) c) \tau-c T(\tau)-\hat{g}]+(1-\tau) \omega+\delta Z^{J}(T(\tau))$,

with $J \in\{I, O\}$. Using the envelope theorem and the first order condition with respect to $\tau^{\prime}$, we can then obtain

$Z_{\tau}^{I}(\tau)=2 \phi(\omega+(1-d) c)-\omega+T_{\tau}(\tau)\left[\begin{array}{c}2(1-\theta)(1-\gamma) c-2 \theta \gamma c \\ -\delta(1-2 \gamma)[2(1-\theta)(\omega+(1-d) c)-\omega]\end{array}\right]$,

if $T(\tau)$ is differentiable. In the redistributive regime, the first order condition with respect to $\tau^{\prime}$ gives

$$
2(1-\theta) c=\delta Z_{\tau}^{I}\left(\tau^{\prime}\right)
$$


so that

$2(1-\theta) c=2 \phi \delta(\omega+(1-d) c)-\delta \omega+\delta T_{\tau}(\tau)\left[\begin{array}{c}2(1-\theta)(1-\gamma) c-2 \theta \gamma c \\ -\delta(1-2 \gamma)[2(1-\theta)(\omega+(1-d) c)-\omega]\end{array}\right]$

A candidate policy function $T(\tau)$ would then have

$T_{\tau}(\tau)=\frac{1}{\delta} \frac{2 \phi \delta(\omega+(1-d) c)-\delta \omega-2(1-\theta) c}{\delta(1-2 \gamma)[2(1-\theta)(\omega+(1-d) c)-\omega]+2 \theta \gamma c-2(1-\theta)(1-\gamma) c} \equiv \Psi$

for $\forall \tau>\tilde{\tau}$.

$\Psi$ is constant, giving a linear function $T(\tau)$. If $\Psi>1$, no function $T(\tau)$ is consistent with equilibrium. However if $\Psi<1$ a policy function $T(\tau)$ as in Figure 3 is consistent with equilibrium.

For all $\tau>\tilde{\tau}$, the policy function $T(\tau)$ maps $\Theta$ to itself and optimality is satisfied by the argument above. $\forall \tau<\tilde{\tau}$, the economy is expected to remain in the common interest regime indefinitely. As the cohesiveness condition does not hold, the Pigouvian steady state cannot be supported, and $T(\tau)$ converges to $\tilde{\tau}$.

For all $\tau<\tilde{\tau}$

$$
Z_{\tau}^{I}(\tau)=U_{\tau}^{I}(\tau)=\alpha V_{g}(g)(\omega+(1-d) c),
$$

thus

$$
\lim _{\tau \nearrow \tilde{\tau}} \delta Z_{\tau}^{I}(\tau)=2(1-\theta) \delta(\omega+(1-d) c)-\omega>2(1-\theta) c=\lim _{\tau \searrow \tilde{\tau}} \delta Z_{\tau}^{I}(\tau)
$$

so that $T(\tilde{\tau})=\tilde{\tau}$ is optimal as well. Optimality conditions are satisfied for all values of $\tau$ and we have confirmed that this is an equilibrium. The economy has a unique and stable steady state at $\tau=\tilde{\tau}$.

This equilibrium is qualitatively different from the redistributive equilibrium that emerges as the limit to a finite horizon economy and bears greater similarity to the weak steady state in the benchmark model.

\subsection{Non-Markov Equilibria}

We have focused on Markov Perfect Equilibria (MPE) in our benchmark model. In this section, we consider a different refinement on subgame perfection that allows history dependence. We outline a model with self-enforcing 


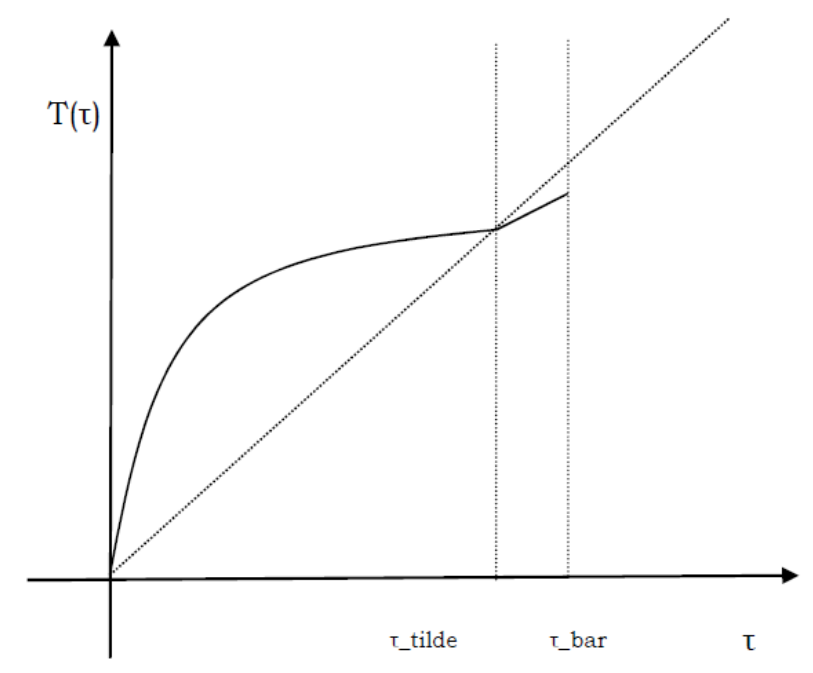

Figure 3: Additional concave equilibrium when stability condition holds.

equilibria supported by punishment strategies. It is well known that the class of such equilibria is large. We follow the recent literature (cf. Acemoglu, Golosov and Tsyvinsky (2008, 2011) and Aguiar and Amador (2011)) and restrict attention to Pareto-efficient equilibria.

In our benchmark model, cooperation is enforced by institutional factors embodied in the parameter $\theta$. In contrast, this appendix studies how cooperation might be endogenously enforced through reputational mechanisms. In this formulation, we allow $\theta$ to be endogenously chosen in each period. Clearly, in any MPE, the optimal choice is $\theta=0 .^{3}$

We outline here some characteristics a reputational equilibrium. We leave a full analysis of this problem for future research. As we are studying efficient subgame perfect equilibria (SPE), the problem can be reformulated as that of a social welfare planner who maximizes the utility of groups $A$ and $B$ subject to participation constraints for both groups. $\psi^{A}$ denotes the Pareto weight assigned by the social planner to group $A$ and $\theta_{s}^{A}$ represents the share of transfers given to group $A$ in period $s$.

When the problem is thus formulated, incumbency no longer plays a

\footnotetext{
${ }^{3}$ In the common interest regime, the incumbent is indifferent between any value $\theta \in$ $\left[0, \frac{1}{2}\right]$.
} 
role, except for the outside options of the two groups. (The "incumbent" is the group that is tempted to deviate from the SPE.) Using the methods introduced by Marcet and Marimon (2011), the planning problem can be formulated recursively as

$$
\left.\begin{array}{rl}
U\left(\tau, \mu^{A}, \mu^{B}\right)=\max _{g, \tau^{\prime}, r, \theta^{A}, \mu^{A^{\prime}, \mu^{B \prime}}} & \left\{\begin{array}{c}
\alpha V(g)+2 r\left[\psi^{A} \theta^{A}+\left(1-\psi^{A}\right)\left(1-\theta^{A}\right)\right]+(1-\tau) \omega \\
+\mu^{A}\left[\alpha V(g)+2 \theta^{A} r+(1-\tau) \omega\right] \\
+\mu^{B}\left[\alpha V(g)+2\left(1-\theta^{A}\right) r+(1-\tau) \omega\right] \\
-\eta^{A} U^{A}(\tau)-\eta^{B} U^{B}(\tau) \\
+\delta U\left(\tau^{\prime}, \mu^{A \prime}, \mu^{B \prime}\right)
\end{array}\right. \\
\text { s.t. }(\omega+(1-d) c) \tau \geq g+r+c \tau^{\prime} \quad \text { and } r>0, \theta^{A} \in[0,1]
\end{array}\right\}
$$

The first line in the maximization problem is the period utility of the social planner, reflecting the weights $\psi^{A}$ and $1-\psi^{A}$ it places on the utility of the two groups. $r$ denotes total transfers. The next three lines are the participation constraints of the two groups with $\eta^{J}$ for $J \in\{A, B\}$ representing the Lagrange multipliers on these constraints. $\mu^{J}$ for $J \in\{A, B\}$ is the shadow value of committing to a policy in period $s$ and evolves recursively according to

$$
\mu_{s}^{J}=\delta \mu_{s-1}^{J}+\eta_{s}^{J} .
$$

The value function $U\left(\tau, \mu^{A}, \mu^{B}\right)$ represents the present value of the social planner having entered a period with fiscal capacity of $\tau$ and promised utilities reflecting shadow prices $\mu^{A}$ and $\mu^{B}$. The identity of the incumbent is an implicit state variable as well. $U^{J}(\tau)$ is the value group $J$ obtains if the incumbent were to deviate from the sustainable plan, i.e. the outside option of group $J$. The identity of the incumbent enters the problem only here. The probability of turnover $\gamma$ also enters this problem only implicitly through the value functions $U^{J}(\tau)$.

We are silent at this point on the specific off-the-equilibrium path punishment and characterize features common to the class of all efficient sustainable SPEs. Recall, however, that for the above problem to be a subgame perfect equilibrium, $U^{A}(\tau)$ and $U^{B}(\tau)$ must in themselves be the value functions for groups $A$ and $B$, respectively, arising from some SPE.

The first order conditions of this maximization problem give

$$
\left(1+\mu^{A}+\mu^{B}\right) \alpha V_{g}(g)=\lambda,
$$

where $\lambda$ is the Lagrange multiplier on the budget constraint. Whenever $r>0$ 
we also have

$$
2\left[\left(\psi^{A}+\mu^{A}\right) \theta^{A}+\left(1-\psi^{A}+\mu^{B}\right)\left(1-\theta^{A}\right)\right]=\lambda,
$$

and for interior choices of $\theta^{A}$ :

$$
\psi^{A}+\mu^{A}=1-\psi^{A}+\mu^{B} .
$$

Finally, an optimal choice of $\tau^{\prime}$ requires

$$
\delta U_{\tau}\left(\tau^{\prime}, \mu^{A \prime}, \mu^{B \prime}\right)=c \lambda .
$$

Applying the envelope theorem to $U^{I}(\tau)$, we derive the following Euler equation:

$$
\alpha c V_{g}(g)=\delta(\omega+(1-d) c) \alpha c V_{g}\left(g^{\prime}\right)-\delta \omega-\delta \frac{\eta^{A \prime} U_{\tau}^{A}\left(\tau^{\prime}\right)+\eta^{B \prime} U_{\tau}^{B}\left(\tau^{\prime}\right)}{1+\mu^{A}+\mu^{B}}
$$

In the redistributive regime, $r>0$ and (18) to (20) combine to give $\alpha V_{g}(g)=1$. The quantity of public goods $g=\hat{g}$ provided when in the redistributive regime is identical to the one that would result from the MPE when $\theta=\frac{1}{2}$.

Note, however, that (20) holds only if $\theta^{A}$ is in the range $\theta^{A} \in(0,1)$. If $\theta^{A}=1$ then (18) to (20) combine to give

$$
\alpha V_{g}(g)=2 \frac{\psi^{A}+\mu^{A}}{1+\mu^{A}+\mu^{B}}
$$

when $r>0$. If $\theta^{A}=0$ then

$$
\alpha V_{g}(g)=2 \frac{1+\mu^{B}-\psi^{A}}{1+\mu^{A}+\mu^{B}}
$$

when $r>0$. In both cases $1<\alpha V_{g}(g)<2$, so that $\hat{g}$ is greater than the level that would emerge in an MPE with $\theta=0$, but less than in an MPE with $\theta=\frac{1}{2}$. Due to cooperation between the factions, public good provision strictly exceeds the level that would arise in the redistributive regime an $\operatorname{MPE}$ (where $\theta=0$ and $\alpha V_{g}(\hat{g})=2$ ).

It is easy to show that a redistributive steady state cannot arise. The model is stochastic, as the identity of the incumbent is a random variable. 
Each time a group $A$ or $B$ is in power, their participation constraint may bind, which gives a strictly positive value of $\eta^{A}$ or $\eta^{B}$ and this alters the value of $\mu^{A}$ or $\mu^{B}$. If the economy remains in the redistributive regime indefinitely, the economy fluctuates based on these exogenous changes in power.

A redistributive steady state would require $\eta^{A}=\eta^{B}=0$, due to (17) and $\lambda=1+\mu^{A}+\mu^{B}$ in every period from (18). (21) then becomes

$$
1+\mu^{A}+\mu^{B}=\lambda=-\frac{\eta^{A} U_{\tau}^{A}(\tau)+\eta^{B} U_{\tau}^{B}(\tau)}{c(1-\delta(1-d))}=0 .
$$

A constant "tightness" of participation constraints would require a decummulation of fiscal capacity over time, which would bring the economy to the common interest regime. The only possibility for a redistributive economy in the long run is one where the values of $\mu^{A}$ and $\mu^{B}$ fluctuate over time.

If an ergodic distribution in the redistributive regime exists, it implies cycles between periods when $\alpha V_{g}(g)=1$, with the share of transfers to each group fluctuating in each period-to ensure their participation-on one hand, and periods where public goods are provided below the first best level, but above what would emerge in an MPE.

The latter occurs if one group remains in power for a large number of periods. Maintaining that group's participation then requires not only that all transfers go to the incumbent, but also that public good provision is eroded to provide a greater amount of total transfers, as implied by (22) and (23).

A common-interest steady state, in contrast, may exist. If it exists, it is at the Pigouvian level of public good provision. In steady state $\eta^{A}=\eta^{B}=0$ and (21) becomes

$$
\alpha V_{g}(g)=\frac{\delta \omega}{\delta \omega-c(1-\delta(1-d))},
$$

giving $g=g^{P}$ : the Pigouvian steady state is attained.

In any period that the participation constraint is binding in the commoninterest regime, the dynamic system in (21) implies a declining path of public good provision and ultimately underprovision of public goods relative to the first best.

The unique sustainable steady state is at the first best: the Pigouvian steady state. Fiscal capacity, public good provision and transfers all fluctuate 
over time if the Pigouvian steady state is not sustainable. Whether the Pigouvian steady state is attainable depends on the punishment SPE implied by $U^{A}(\tau)$ and $U^{B}(\tau)$.

We conclude that history-dependent equilibria may be qualitatively different from the Markov Perfect Equilibrium. First, a Pigouvian steady state can potentially be supported even if there are no exogenous institutional constraints on redistribution. Second, redistributive and weak steady states do not exist, although ergodic distributions within these regimes do potentially exist. But the three types of state emerging from our benchmark model do appear arise along equilibrium paths of the sustainable equilibrium: a common-interest state, a redistributive state, and a weak state with underprovision of public goods. We leave to future research a full characterization of sustainable equilibria in this model.

\section{References}

[1] Aguiar, Mark and Manuel Amador, 2011, "Growth in the Shadow of Expropriation," The Quarterly Journal of Economics, 26(2), 651-697.

[2] Marcet, Albert and Ramon Marimon, 2011, "Recursive Contracts," CEP Discussion Papers dp1055, Centre for Economic Performance, LSE. 